\title{
Inicio de programa para resolver problemas sencillos
}

\section{Assumpta Cervelló * Josefina Sabaté}

\author{
"Escola Gavina» de la OS AS. Barcelona
}

\section{INTRODUCCION .}

El planteamiento inicial que nos ha llevado a realizar este trabajo ha sido el de dar respuesta a una serie de interrogantes que se nos planteaban a la hora de enseñar a nuestros alumnos a resolver problemas.

Hemos de hacer notar la escasez bibliográfiça, castellana y catalana, sobre el tẹma, dirigida a maestros y educadores. Así, nuestras premisas, básicamente, parten de la propia experiencia.

Ya iniciado el planteamiento de este trabajo nos llegó un libro de Gaston Mialaret (1977) editado en lengua castellana, traducción del original francés de 1967 .

Este autor hace un análisis bastante real de los tipos de errores que suelen tener los alumnos cuando resuelven problemas, pero no nos ha sido útil a la hora de encontrar soluciones para resolverlos. Nosotros pretendemos elaborar una programación coherente para enseñar a resolver problemas en nuestro centro. Esto implica establecer unos objetivos a alcanzar por cada grupo de alumnos según su nivel de desarrollo y el procedimiento que hay que utilizar para conseguirlo.

Hemos considerado la resolución de problemas como un aprendizaje complejo donde inciden componentes de tipo aritmético juntamen-

\section{Colaboraciones}

Agradecemos a los profesionales:

Boix i Puig, Montserrat

Casagran i Borrell, Xavier

Clusellas i Casals, Mohtserrat

Garcia i Trepat, M.a Pilar

Riera i Euras, Ramón

que hayan contribuido en la confección del material, en la puesta en práctica del procedimiento $y$ en las observaciones en las aulas.

* Dirección de las autoras: Escola Gavina. Fraga, 2. Barcelona-2o. 
te con otros de lenguaje y hemos supuesto que, enseñando a los alumnos a encadenar las tareas que han de realizar para resolver un problema, mejorarian su aprendizaje.

\section{OBJETIVOS}

Es evidente que en la «resolución de problemas» intervienen, desde la diversidad de los componentes de tipo matemático (numeración que se utiliza en los datos y operaciones a efectuar) a las de lenguaje ("formato" o manera de explicar la situación) que dependen del vocabulario utilizado, de la estructura morfo-sintáctica, de la secuenciación temporal, de la narrativa, etcétera.

Hemos preferido establecer unas condiciones mínimas para iniciar la definición de los objetivos, que serían:

\section{Respecto al lenguaje:}

a) El vocabulario será conocido y familiar al niño.

b) Los modos verbales utilizados serán el indicativo y el infinitivo.

c) Las situaciones narradas se referirán a la vida cotidiana de los alumnos.

d) Habrá una secuenciación continua de la narrativa, de forma que la lectura del texto diga al niño qué es lo que está pasando, de una forma temporalmente ordenada, en la que aparezcan en un primer momento los datos y después la pregunta.

A este tipo de problema con una disposición secuencial del texto se le llama también "problema por etapas» (Mialaret, G., 1977, pág. 61).

\section{Respecto a los componentes de matemáticas:}

a) El alumno estará inịciado en el aprendizaje, copia y lectura de los números que aparecen en los datos del problema.

b) El problema será de una sola operación.

c) El tipo de operaciones a resolver será: suma, resta, multiplicación y división.

d) El tipo de resta que se ha de realizar a partir del enunciado será el controlado por las palabras que significan: «quitar» (perder, gastar, regalar, etcétera) y no aquellas en que la diferencia sea el resultado de una comparación (cuánto falta para..., cuánto es más grande, etcétera...).

Esta norma sólo será válida para los dos primeros niveles de etapa escolar.

\section{Ler Nivel}

Dado un texto que el profesor leerá en voz alta, teniendo en cuenta las componentes de lenguaje antes citadas y con datos numéricos en enteros $\leqslant$ al 100 , que impliquen una suma de dos sumandos llevando o sin llevar, o una resta sin llevar, el alumno dará por escrito el 
planteamiento, la operación hecha sin errores y la respuesta numéricamente correcta escribiendo el tipo de unidades o especie del resultado.

\section{2. $\quad$ Nivel}

Dado, un texto escrito del tipo antes citado, que el alumno tendrá en una hoja de papel con datos numéricos en enteros $\leq$ al 1.000 , que impliquen una suma de dos o tres sumandos llevando o sin llevar, o una resta llevando o sin llevar, el alumno dará por escrito el planteamiento, la operación hecha sin errores y la respuesta numérica y gramaticalmente correcta, especificando el tipo y especie del resultado numérico.

\section{3. ${ }^{\text {er }}$ Nivel}

Dado un texto escrito del tipo antes citado, que el alumno tendrá en una hoja de papel con datos numéricos en enteros $\leqslant 1.000 .000$, y que comporten una operación de sumar, restar o multiplicar, el alumno dará por escrito el planteamiento, la operación realizada sin error y la respuesta numéricamente y gramaticalmente correcta.

\section{$4 \cdot^{\circ}$ Nivel}

Dado un texto escrito del tipo antes citado, que el alumno tendrá en una hoja de papel con datos numéricos en enteros que pueden ser superiores a 1.000 .000 y que supongan una operación de sumar, restar, multiplicar o dividir, el alumno dará por escrito el planteamiento, la operación realizada correctamente y la respuesta numérica y gramaticalmente correcta.

\section{$50^{\circ}$ Nivel}

Dado un texto escrito del tipo antes citado, que el alumno tendrá escrito en una hoja de papel con datos numéricos que pueden ser enteros superiores a 1.000 .000 , o decimales que impliquen una operación de sumar, restar, multiplicar o dividir, el alumno dará por escrito el planteamiento, la operación realizada correctamente y la respuesta numérica y gramatical correcta.

\section{Análisis de tareas}

Se ha hecho exclusivamente el correspondiente a los problemas que constituyen el objetivo de $10^{\circ}$ y $2 .^{\circ}$ nivel.

Analizando las tareas que puede comportar al alumno "resolver» este tipo de problemas, hemos encontrado las siguientes, que hemos agrupado en dos grandes bloques según hagan referencia a la comprensión del texto o a la resolución propiamente dicha.

\section{Comprensión del texto:}

I. Identificación de los datos (por medio de preguntas).

2. Identificación de la cuestión "a resolver» (por medio de preguntas sobre la pregunta del problema). 
3. Decir cuál es el cambio cuantitativo que supone la aparición secuencial de los datos desde la situación inicial hasta la pregunta.

4. Decir la operación matemática que corresponde a este cambio cuantitativo.

5. Escribir el planteamiento numérico de la operación.

6. Efectuar la operación planteada.

7. Dar la respuesta por escrito a la pregunta.

Hemos supuesto que el encadenamiento progresivo de estas tareas llevaría al alumno a alcanzar el objetivo planteado y considerando que el profesor, a través de controles verbales, puede enseñar a sus alumnos a encadenarlas, hemos propuesto el siguiente procedimiento, con retirada progresiva de soportes que llevamos a cabo en cinco fases.

A continuación las especificamos aplicadas a un problema concreto.

\section{INICIO DEL PROCEDIMIENTO}

\section{Fase A}

\section{Comprensión del texto}

i. El profesor leerá en voz alta el texto del problema, escribiendo al mismo tiempo los datos numéricos.

"La gata de Joaquín ha tenido s gatitos y una vecina se ha llevado 3 a su casa. ¿Cuántos gatitos le quedan a la gata?» Escribirá: s gatitos

$$
3 \text { gatitos }
$$

2. Preguntará sobre la situación inicial de manera que la respuesta sea numérica. «¿Cuántos gatitos ha tenido la gata de Joaquín?»

3. Preguntará sobre la situación posterior (introducción del nuevo dato) de manera que la respuesta sea numérica. "¿Cuántos gatitos se ha llevado a su casa la vecina?»

4. Preguntará:

"¿Qué te pregunta el problema?»

\section{Resolución}

5. Preguntará sobre el cambio cuantitativo que supone la aparición secuencial de los datos.

«Si la gata tenía, gatitos y la vecina se ha llevado 3, cle quedarán más o menos?»

6. Aparejará el cambio cuantitativo con la operación matemática que correponda. Lo hará utilizando una fórmula verbal parecida a ésta:

«Si le quedan menos gatos, para saber cuántos le quedan tendremos que restar.»

7. Planteará numéricamente en la pizarra lo que ha pasado, 
aparejándolo con la palabra "planteamiento» y leyendo en v'sz alta lo que escribe.

Dirá a los alumnos que lo copien.

«Ir escribiendo con números lo que ha pasado. Ir planteando el problema.»

"s gatitos que tenía la gata menos 3 que se lleva la vecina igual..."

$$
5-3=\ldots
$$

«Copiarlo».

8. Preguntará el resultado de la operación y la escribirá en la pizarra. "¿Cuántos son cinco menos tres?»"

«Cinco menos tres son dos».

9. Preguntará cuál es la pregunta del problema. "¿Qué nos pregunta el problema?»

10. Dará respuesta oralmente, escribiendo al mismo tiempo en la pizarra una $\mathrm{R}$ mayúscula, la cifra numérica y la clase de unidades. Después dirá a los alumnos que la copien.

«La respuesta es dos gatitos».

$\mathrm{R}: 2$ gatitos.

\section{Fase B}

Comprensión del texto

1-2-3-4-igual que en la fase A.

\section{Resolución}

5. Igual que en la fase $A$.

6. Preguntará la operación que hay que hacer:

“¿Qué operación tienes que hacer?»

7. Dirá:

"Escribe el planteamiento.»

8. Dirá:

«Haz la operación.»

9. Igual que en la fase $A$.

10. Preguntará:

«¿Cuál es la respuesta?»

\section{Fase C}

Comprensión del texto

1. El profesor escribirá el texto en la pizarra y lo leerá en voz alta él mismo o lo hará leer a los niños que lean bien. 
2. Preguntará

"¿Qué dice, en primer lugar, el problema?»

3. Preguntará:

"¿Qué ha pasado después?»

4. Preguntará qué hace falta saber.

\section{Resolución}

5 y 6 igual que en $B$.

7. Preguntará qué es lo que se ha de hacer y dirá que lo hagan.

8. Preguntará:

«¿Cuál es la respuesta?» y la escribirá de una forma completa en la pizarra.

R: a la gata le quedan 2 gatitos y dirá: «copiad la respuesta de esta manera».

\section{Fase D}

Comprension del texto

1. El profesor escribirá el texto en la pizarra y dirá a los alumnos que lo copien y lo lean en voz baja.

\section{Resolucion}

2. Preguntará:

«¿Qué operación hay que hacer?»

3. Dirá:

«Hacer todo lo que queda.»

\section{Fase E}

Esta fase corresponde a la situación de test final, con la diferencia que en este test, el texto se le da ya escrito y en la fase E, en cambio, lo copia de una ficha.

\section{Situación y material}

El procedimiento se trabaja en situación de grupo.

Los alumnos están en su aula en donde hay una pizarra grande, mesas y sillas individuales que pueden ser colocadas como convenga.

El material utilizado por los alumnos desde la primera sesión es una hoja de papel blanco, un lápiz y una goma.

En determinadas sesiones (y sobre todo al principio) había un observador en la clase que tomaba nota de los hechos más destacables y registraba en la hoja correspondiente las respuestas de los alumnos. 
El profesor tenía el procedimiento y lo iba siguiendo.

Después de las sesiones, el observador comentaba los hechos a destacar con el profesor del aula y quedaban de acuerdo en la elaboración y uso del material para la sesión siguiente.

Los problemas que se trabajaban cran preparados previamente por el profesor y/o el observador.

\section{Conductas previas}

Los aprendizajes académicos que hemos considerado necesarios al iniciar el procedimiento son los siguientes:

I. Leer los números hasta el 9 .

2. Copiar de la pizarra correctamente.

\section{Sujetos y tratamiento}

El procedimiento ha sido aplicado a una población formada por dos grupos de alumnos que presentan las siguientes características:

\section{Grupo I}

- Formado por 13 niños y niñas de edades comprendidas entre 8 y i I añós (sólo hay un sujeto de 1 I años), 4 de los cuales realizan la escolaridad en catalán.

- Ios C. I. de estos alumnos, según la escala de WISC, oscila entre 60 y 80 , aunque uno de ellos tiene un 50.

- Las sesiones son de una hora semanal y durante las mismas hay, normalmente, dos educadores en el aula.

- Los textos son leídos en catalán y castellano y a cada alumno se le pregunta individualmente en su propia lengua.

- Desde que se inició la puesta en marcha del procedimiento, estos alumnos han recibido unas i 8 sesiones colectivas $y$ actualmente se encuentran en la fase B del mismo, es decir, todavia no han llegado al objetivo del Primer Nivel.

Grupo 2

Está formado por 13 niños y niñas, I I de los cuales tienen edades comprendidas entre 9 y I 1 años, habiendo uno que tiene 12 y otro que tiene 8.

- Tres de estos alumnos realizan la escolaridad en catalán.

- El C. I., según la escala de WISC, de i I de estos alumnos oscila entre 66 y 87 , habiendo uno con so y otro con 103. Este último será integrado en una escuela normal el curso próximo.

- Las sesiones son de media hora semanal y durante las mismas hay dos educadores en el aula.

- Los textos de los problemas son escritos en la pizarra en catalán y castellano sucesivamente $y$, cuando los textos en ambas formas están en la pizarra, son leídos por el profesor en voz alta 
para todos los alumnos. Las preguntas dirigidas a cada uno se formulan en su. propia lengua contestando ellos de la misma forma.

- Desde que se inició la enseñanza de los problemas, los alumnos han tenido 14 sesiones. Actualmente están en la fase $E$.

- El reforzador utilizado en ambos grupos es el social.

\section{CONCLUSIONES}

A pesar de que nuestro trabajo, iniciado hace poco, no haya podido tener un tratamiento de diseño experimental como hubiésemos querido $y$ que probablemente intentaremos en un futuro, valoramos positivamente:

a) El esfuerzo realizado para el inicio de una sistematización en la enseñanza de una tarea compleja y en la que encontramos una incidencia tan elevada de fracaso escolar.

b) La influencia favorable que la aplicación del procedimiento ha tenido, aunque en fase de revisión, sobre el aprendizaje de los niños, ya que a pesar de no tener un registro de línea base sabemos y nos lo han demostrado los registros de las sesiones que conservamos, que nuestros alumnos no habían recibido nunca un entrenamiento en la tarea de resolver problemas.

c) La impresión que los profesionales hemos tenido, por la buena acogida de los alumnos, sobre todo los del grupo I, de que se lo pasaban bien en la clase de matemáticas, sin necesidad de grandes montajes de material u otros instrumentos y utilizando el reforzador social únicamente.

d) La generalización que en algún caso se ha producido de los aprendizajes realizados en la clase de problemas:

El niño que repetía en voz alta nuestro procedimiento cuando le pedían, mientras le pasaban la prueba del WISC, la resolución de los ítems de cálculo.

\section{Referencias}

Mialaret, G.: Las matemáticas, cómo se aprenden, cómo se enseñan. Madrid, Pablo del Río-Editor, 1977. 